\title{
Maternal fish oil supplementation during lactation is associated with reduced height at 13 years of age and higher blood pressure in boys only
}

\author{
L. Lauritzen ${ }^{1 *}$, S. E. Eriksen ${ }^{1,2}$, M. F. Hjorth ${ }^{1}$, M. S. Nielsen ${ }^{1}$, S. F. Olsen ${ }^{3}$, K. D. Stark ${ }^{4}$, K. F. Michaelsen ${ }^{1}$ \\ and C. T. Damsgaard ${ }^{1}$ \\ ${ }^{1}$ Department of Nutrition, Exercise and Sports, University of Copenhagen, 1958 C Frederiksberg, Denmark \\ ${ }^{2}$ University College Metropol, 1126 K Copenhagen, Denmark \\ ${ }^{3}$ SSI, 2300 S Copenhagen, Denmark \\ ${ }^{4}$ Department of Kinesiology, University of Waterloo, Waterloo, ON, Canada, N2L 3G1 \\ (Submitted 28 October 2016 - Final revision received 18 November 2016 - Accepted 24 November 2016 - First published online 9 January 2017)
}

\section{Abstract}

Dietary long-chain n-3 PUFA ( $n$-3 LCPUFA) in infancy may have long-term effects on lifestyle disease risk. The present follow-up study investigated whether maternal fish oil (FO) supplementation during lactation affected growth and blood pressure in adolescents and whether the effects differed between boys and girls. Mother-infant pairs ( $n$ 103) completed a randomised controlled trial with FO ( $1.5 \mathrm{~g} / \mathrm{d} n-3 \mathrm{LCPUFA}$ ) or olive oil (OO) supplements during the first 4 months of lactation; forty-seven mother-infant pairs with high fish intake were followed-up for 4 months as the reference group. We also followed-up 100 children with assessment of growth, blood pressure, diet by FFQ and physical activity by 7-d accelerometry at 13.5 (SD 0.4 ) years of age. Dried whole-blood fatty acid composition was analysed in a subgroup ( $n$ 49). At 13 years of age, whole-blood $n$-3 LCPUFA, diet, physical activity and body composition did not differ between the three groups. The children from the FO group were $3.4(95 \% \mathrm{CI} 0 \cdot 2,6 \cdot 6) \mathrm{cm}$ shorter $(P=0.035)$ than those from the OO group, and tended to have less advanced puberty $(P=0.068)$, which explained the difference in height. There was a sex-specific effect on diastolic blood pressure $\left(P_{\text {sex } \times \text { group }}=0.020\right)$, which was driven by a $3.9(95 \%$ CI $0.2,7.5) \mathrm{mmHg}$ higher diastolic blood pressure in the FO compared with the OO group among boys only $(P=0 \cdot 041)$. Our results indicate that early $n-3$ LCPUFA intake may reduce height in early adolescence due to a delay in pubertal maturation and increase blood pressure specifically in boys, thereby tending to counteract existing sex differences.

\section{Key words: n-3 Long-chain PUFA: Puberty: Growth: Programming: Health}

Dietary long-chain $n$-3 PUFA ( $n$-3 LCPUFA) are required for normal child development ${ }^{(1)}$, and intake of $n$-3 LCPUFA during early infancy has also been proposed to have long-term effects on IQ and cardiovascular health ${ }^{(1,2)}$. During infancy, breast milk is an important source of $n-3$ LCPUFA, specifically $\mathrm{DHA}^{(1,3)}$, and breast-feeding compared with formula feeding has been associated with long-term favourable effects on $\mathrm{BMI}^{(4)}$ and blood pressure ${ }^{(5,6)}$. These effects could in part be due to the relatively high content of DHA in breast milk, as infant formula until recently did not contain LCPUFA. However, a few studies have investigated the potential effects of early $n-3$ LCPUFA supplementation on later health.

Animal studies have shown that $n-3$ LCPUFA can act as a nutritional programming factor in prenatal and postnatal life, affecting the proliferation and differentiation of pre-adipocytes and preventing excessive adipose tissue development ${ }^{(7,8)}$. The few studies that have examined the effects of early $n$ - 3 LCPUFA supply through infant formula or breast milk on children's body composition later in life show conflicting results ${ }^{(9-12)}$. Furthermore, it is difficult to interpret whether the observed effects are indeed beneficial as body composition varies with decreasing BMI from about 1 year of age and then rebounds at 4-7 years of age from where it increases towards adult levels ${ }^{(13)}$. Thus, some of the inconsistency could be due to fluctuations in body composition during growth. Pedersen et $a l^{(14)}$ observed that high DHA concentration in breast milk was associated with a delay in the timing of adiposity rebound, most strongly in girls, which could be protective against later obesity. It may therefore be relevant to assess potential effects later in childhood.

In rodents, perinatal intake of $n-3$ PUFA has been shown to reduce blood pressure later in life ${ }^{(15,16)}$. Human intervention studies have also indicated that early intake of $n$ - 3 LCPUFA can affect later blood pressure, but the results are inconsistent. One study found that addition of LCPUFA to infant formula was associated with a lower mean arterial blood pressure (MAP) and diastolic blood pressure (DBP) at 6 years of age ${ }^{(17)}$. In contrast, no

Abbreviations: DBP, diastolic blood pressure; FO, fish oil; $n$-3 LCPUFA, long-chain $n$-3 PUFA; MAP, mean arterial blood pressure; OO, olive oil.

* Corresponding author: Associate Professor L. Lauritzen, fax +45 3533 2483, email 11@nexs.ku.dk 
difference in blood pressure at 8 years of age was found in a randomised controlled trial where children received fish oil (FO) supplements from the time they stopped breast-feeding to age 5 years ${ }^{(18)}$. Another follow-up study showed no difference in blood pressure among the 19-year-old offspring of mothers who were randomly allocated to either $n$-3 LCPUFA supplementation or control during the last trimester of pregnancy ${ }^{(19)}$. In our FO supplementation trial during lactation ${ }^{(20)}$, we found higher blood pressure at 7 years of age in sons of mothers who were supplemented with FO, but no effect among girls ${ }^{(10)}$. In another previous study, we have also observed sex-specific effects of $n-3$ LCPUFA supplementation on blood pressure in children ${ }^{(21)}$ and sex-specific associations between $n$-3 LCPUFA status and blood pressure $^{(22,23)}$. Sex differences in response to $n$-3 LCPUFA intake during infancy have also been observed by others with respect to cognitive outcomes ${ }^{(24)}$, but have not been taken into account in most studies with focus on growth or in studies with long-term follow-up.

Therefore, the aim of the present follow-up study was to explore the effect of maternal FO supplementation during lactation on growth, body composition and blood pressure in the offspring at 13 years of age, in order to investigate whether the previous observed associations were transient or withstanding. We furthermore aimed to examine potential effect modifications by sex.

\section{Methods}

\section{Study design, participants and intervention}

The present 13-year follow-up study was based on a doubleblinded randomised controlled trial in lactating mothers ${ }^{(20)}$. We conducted three previous follow-up studies among the offspring - at age 9 months ${ }^{(20)}, 2.5$ years $^{(25)}$ and 7 years ${ }^{(10)}$. The original trial and the previous and present follow-up studies are registered at ClinicalTrials.gov (NCT00266305), approved by the Committees on Biomedical Research Ethics for the Capital Region of Denmark (KF 01-300/98, KF 01-183/01, KF 11321572 and H-3-2012-150), and all custody holders of each child gave informed written consent for participation.

The original trial recruited healthy, Danish pregnant women from the Danish National Birth Cohort (DNBC) ${ }^{(26)}$ during December 1998 to November 1999(20). Women were selected from the DNBC on the basis of their place of residence (the greater Copenhagen area) and their self-reported $n-3$ LCPUFA intake from fish. Women with an intake of $n-3$ LCPUFA from fish below the population median $(<0.40 \mathrm{~g} / \mathrm{d}$ of $n-3$ LCPUFA, consuming on average 12.3 ( $\mathrm{SD} 8 \cdot 2) \mathrm{g} / \mathrm{d}$ of fish) were randomly allocated to two groups - FO and olive oil (OO). Those with an intake above the 75 th percentile ( $>0.82 \mathrm{~g} / \mathrm{d}$ of $n$ - 3 LCPUFA, consuming on average 55 (SD 27) $\mathrm{g} / \mathrm{d}$ of fish) were recruited to a high-fish (HF) reference group (see trial flow in Fig. 1).

Randomisation was performed within 9 (SD 3) d after delivery, and the mothers received supplements during the first 4 months of lactation. The oil supplements were given in muesli bars containing de-odourised microencapsulated oil powder, which contained $4.5 \mathrm{~g} / \mathrm{d}$ of FO and supplied $1.5 \mathrm{~g} / \mathrm{d} n-3$ LCPUFA $(0.6 \mathrm{~g}$ $\mathrm{EPA}+0.8 \mathrm{~g}$ DHA) or $4.5 \mathrm{~g} / \mathrm{d}$ of OO. Self-reported compliance, expressed as the percentage of muesli bars consumed relative to the intended dose, was on average $88 \%$ in both groups ${ }^{(20)}$.
A total of 107 mothers (87\%) reported to have exclusively breast-fed their infants during all 4 months of intervention, which is common in Denmark. Women who had not exclusively breast-fed their child during the 4-month intervention period were not excluded from the trial, but the degree of breast-feeding (in percentage of the child's energy intake) was estimated from the amount of formula and complementary food ingested as described in the study by Lauritzen et $a l^{(20)}$. Compliance was furthermore confirmed by GC fatty acid analysis of maternal erythrocytes and breast milk before the intervention (at 36.4 (SD 1.5) weeks of gestation and 9 (SD 3) d after birth, respectively) and during the intervention (at 2 and 4 months for breast milk and at 4 months for maternal erythrocytes). Infant erythrocyte fatty acid composition was also determined at the end of the intervention (4 month) ${ }^{(20)}$. Investigators and families were blinded to the randomisation during the trial, but the code was broken for all parties after the youngest child's first birthday (January 2001).

Infant growth was assessed twice during the supplementation period, at age 2 and 4 months. Birth data on length and height were collected from hospital journals. The infant's head circumference was measured at the first visit after randomisation that is, 9 ( $\mathrm{SD} 3$ ) d after delivery. Anthropometric measurements were performed at the follow-up examinations at age 9 months, $2 \cdot 5$ years and 7 years, as previously described ${ }^{(10,20,25)}$. Background information regarding parental education, health, anthropometry, etc. was collected via questionnaires ${ }^{(20)}$. Data on maternal education were collected by Official Danish Classification of Educations from 1994 in eight categories from $1=$ primary school to $8=\mathrm{PhD}$. As there were very few subjects in the extreme categories, the education variable was re-coded to four categories representing a high school education or less, 13-14 years of education, 15-16 years and >17 years of education (equivalent to a University master degree).

\section{3-Year follow-up examination}

The 140 mothers, who had not actively withdrawn their participation from the study, were invited to participate in the present follow-up study with their children. In total, 100 children participated in the present 13-year follow-up study. Among the forty mothers who did not participate, twenty mothers did not respond to a postal letter or telephone calls, eight no longer resided in the greater Copenhagen area, eleven did not wish to participate because of personal reasons and one child had passed away. Blood sample collection had not been ethically approved at the initiation of the follow-up study, and was thus only performed in forty-nine the children.

The follow-up examination took place either at Department of Nutrition, Exercise and Sports, University of Copenhagen, or at home of the family; two investigators, who were blinded to the group allocation, performed the measurements on all children. Height was measured twice to the nearest $0 \cdot 1 \mathrm{~cm}$ using a portable stadiometer (Leicester Height Measure; Child Growth Foundation). Head, waist and hip circumferences were assessed in triplicate with a non-stretchable measuring tape to the nearest $\mathrm{mm}$. Triceps and subscapular skinfold thicknesses were assessed in triplicate using a Harpenden skinfold caliper with a resolution 
of $0.1 \mathrm{~mm}$ (CMS Weighing Equipment; Baty International). The lack of inter-investigator differences was verified in the triceps skinfold assessments by comparison in ten subjects $(P=0.598)$. Body weight (to the nearest $\mathrm{g}$ ) and fat percentage were assessed twice by bioimpedance on a body fat monitor scale (Omron BF511; Mediq A/S). The assessed body fat percentage correlated with that calculated from the sum of triceps and subscapular skinfolds on the basis of the methods of Slaughter et al. ${ }^{(27)}$ ( $r$ 0.831, $P<0.001, n$ 99). For all anthropometric measures, we used the mean of the duplicates or triplicates. Fat mass index was calculated on the basis of the bioimpedance assessment as follows: body fat $\% \times$ body mass $(\mathrm{kg}) /$ height $^{2}\left(\mathrm{~m}^{2}\right)$.

Blood pressure was measured in triplicate with an automated device (Boso-medicus Prestige unit; Bosch Sohn). The child was asked to rest for $10 \mathrm{~min}$ in the supine position before the measurements, which were taken with an interval of approximately $2 \mathrm{~min}$. Blood pressure was measured on the non-dominant arm with a cuff designed for small $(12-22 \mathrm{~cm})$ or medium $(22-26 \mathrm{~cm})$ arm circumferences, whichever fitted the child. From these assessments, we calculated the mean of the second and third measurement of systolic blood pressure (SBP) and DBP. MAP was calculated as $\mathrm{DBP}+1 / 3 \times(\mathrm{SDP}-\mathrm{DBP})$.

Pubertal status was self-assessed using Tanner Scales ${ }^{(28)}$. The Tanner Scales consist of line drawings that portray different stages of pubertal development rated on a five-point scale (I, II, III, IV, V), where stage I is classified as pre-pubertal and stage $\mathrm{V}$ as fully matured. The boys were asked to identify their development on the basis of their genital development and pubic hair growth, whereas the girls were asked to identify their development on the basis of breast development. The girls were furthermore asked to record whether their menarche had occurred and the date of its first occurrence.

Fingertip-prick blood samples were collected from $49 \%$ of the children who completed the 13-year follow-up and analysed for fatty acid composition by GC. The blood samples were collected on chromatography paper (Whatman Ltd) prepared with $50 \mu \mathrm{g}$ of 2,6-di-tert-butyl-4-methylphenol (butylated hydroxytoluene; Sigma-Aldrich), allowed to air dry and were stored at $4^{\circ} \mathrm{C}^{(29)}$ until they were shipped to the Department of Kinesiology at University of Waterloo, Canada. The samples were stored at $-80^{\circ} \mathrm{C}$ until analysis using a high-throughput GC method ${ }^{(29)}$. In brief, fatty acid methyl esters were prepared from the whole-blood samples using direct transesterification with the addition of $1 \mathrm{ml}$ of $14 \%$ $\mathrm{BF}_{3}$ in methanol, $300 \mu \mathrm{l}$ of hexane and $3 \mu \mathrm{g}$ of an internal standard (22:3n-3 methyl ester; Nu-Check Prep) followed by heating at $95^{\circ} \mathrm{C}$ for $1 \mathrm{~h}$ on a heating block. The fatty acid methyl esters in hexane were then collected and analysed on a Varian 3900 GC equipped with a DB-FFAP $15 \mathrm{~m} \times 0.10 \mathrm{~mm}$ i.d. $\times 0 \cdot 10-\mu \mathrm{m}$ film thickness, nitro-terephthalic acid-modified, polyethylene glycol capillary column ( $\mathrm{J} \& \mathrm{~W}$ Scientific from Agilent Technologies) with hydrogen as the carrier gas with settings as described in detail elsewhere ${ }^{(29)}$. More than $90 \%$ of the fatty acid peaks in the chromatograms were identified, and individual fatty acids are presented as weight percentage of total fatty acids (FA\%).

By the end of the 13-year examination period, the mother and child received oral and written instructions on using a validated web-based FFQ dietary assessment tool ${ }^{(30)}$ to record the child's dietary intake during the last month. This tool has been developed specifically for adolescents, and asks about 145 commonly eaten foods, dishes and drinks consumed by Danish adolescents for breakfast, lunch and dinner, respectively. Frequency scales were used depending on the food item. Calculations of intake in $\mathrm{g} / \mathrm{d}$ were computed on the basis of standard portion sizes and frequencies ranging from 'did not drink/consume the last month' to 'two to four times or more per day' and were computed into times. The average daily energy intake and distribution of macronutrients (in $\mathrm{g} / \mathrm{d}$ and percentage of the total energy intake) were calculated from the FFQ using FoodCalc version 1.3 combined with the Danish Food Table.

Physical activity was assessed using a tri-axis accelerometer (ActiGraph $^{\mathrm{TM}}$ GT3X+; ActiGraph Corp.). The children were instructed to wear the device tightly at the right hip in an elastic belt for 7 consecutive days, and to remove it during night-time sleep, showering and swimming. Data were re-integrated to 60-s epochs and analysed using ActiLife (version 6; ActiGraph). Nonwear time was defined as $20 \mathrm{~min}$ of consecutive zeros using vector magnitude of the three axes. Valid physical activity recordings were defined as consecutive wear time periods of $\geq 1 \mathrm{~h}$ duration and a total wear time of $\geq 10 \mathrm{~h} / \mathrm{d}$ for $\geq 3 \mathrm{~d}$. Accordingly, eighty-eight children had valid physical activity recordings with a mean duration of 6.7 (SD 1.7) d. Only four children did not remove the accelerometer on one or more nights; in these cases, sleep was removed, on the basis of visual inspection of the individual actograms, as the difference between time when activity stopped in the evening and time when activity resumed in the morning. Total physical activity (counts per min) was expressed as a vector magnitude of the total tri-axial counts from monitor wear time divided by monitor wear time.

\section{Statistics}

Numbers in the text are means unless otherwise stated. Parental and infant characteristics of participating and non-participating children were compared using the unpaired $t$ test (continuous variables) and the $\chi^{2}$ test (categorical variables). Anthropometry, body composition and growth as well as macronutrient intake, fatty acid composition of whole blood and total physical activity in the two randomised groups (FO and OO) were compared in ANCOVA including age and sex as fixed effects. All models were initially tested for sex $\times$ group interaction, and if the $P$ value of the interaction was $<0 \cdot 10$ the analysis was performed separately for boys and girls. Additional analyses were performed after adjusting for potential confounders: maternal education and mean parental height that tended to differ between the randomised groups and potential mediators: puberty in the models of all the anthropometric measures and height in the models of body weight, body composition and blood pressure. The non-randomised HF reference group was not included in the ANCOVA models, but is shown in the tables for comparison. Potential differences in categorical variables (such as pubertal stage) between the two randomised groups were tested using logistic regression analysis adjusted for age and sex; the menarcheal status of the girls in the two groups was compared using the $\chi^{2}$ test.

When outcome variables differed significantly between the FO and OO group in ANCOVA, dose-response analyses were 
performed by regression analysis with maternal erythrocyte DHA in the 4th month of lactation as the independent variable and including children both from the two randomised groups and from the high-fish reference group. If the ANCOVA model showed sex $\times$ group interaction, the corresponding dose-response regression analysis was also performed separately for each sex, and the dose-response models included the same confounders and mediators as the fully adjusted ANCOVA models. In addition, the dose-response models were adjusted for the degree of breast-feeding, as breast-feeding would be a prerequisite for an effect of maternal n-3 LCPUFA status on the child. Data analysis was performed using STATA/IC version 12.1 for Mac (64-bit Intel) and SPSS version 22 , and $P<0.05$ was considered statistically significant. A post boc power calculation showed that with a mean of thirty-two subjects in each of the two randomised groups, this study was powered $(\alpha=0.05$ and $\beta=0.80)$ to show an unadjusted difference of about $0 \cdot 7 \times \mathrm{sD}$.

\section{Results}

\section{Children's characteristics}

Fig. 1 gives an overview of the study flow with recruitment, randomisation, drop out and attrition in the present follow-up study. In total, 100 children (70\% of the invited children) participated in the 13-year follow-up (Table 1), including sixty-four from the two randomised groups ( 58 and $70 \%$ from the FO and OO group, respectively). Participation rate did not differ between the two randomised groups $(P=0 \cdot 223)$, but there was a skewed sex distribution in the FO group, which reflects a skew in the original randomisation. With respect to the characteristics as listed in Table 1, the 100 children who participated in the 13-year follow-up did not differ from those not participating, except for a longer duration of breast-feeding in the mothers of the participating children (9.2 (SD 4.3) v. 7.4 (SD 4.5) months, $P=0 \cdot 049)$. The forty-nine children who provided blood spot samples for fatty acid analysis were slightly older (13.7 (SD 0.4) $v$. 13.3 (sD 0.3 ) years, $P<0.001$ ) than those who were not blood sampled, but this did not differ with respect to dietary macronutrient contribution, maternal education or sex distribution (data not shown).

The two randomised groups did not differ with respect to the children's energy intake, dietary macronutrient distribution or total physical activity, and no sex $\times$ group interactions were observed for any of these variables (online Supplementary Table S1). Moreover, no differences were observed in the content of the major fatty acids in whole blood at 13 years of age (online Supplementary Table S2).

\section{Growth and pubertal stage}

The children from the FO group were $3.7(95 \%$ CI $0.3,7 \cdot 1) \mathrm{cm}$ shorter than those from the SO group at 13 years of age (Table 2). This difference did not change by adjustment for mean parental height (Table 2). However, there was a tendency for lower puberty scores in the children of the FO compared with the OO group at 13 years $(P=0 \cdot 068$, logistic regression with adjustment for age and sex) (Fig. 2), and the difference in height seemed to be driven by this, as the height difference

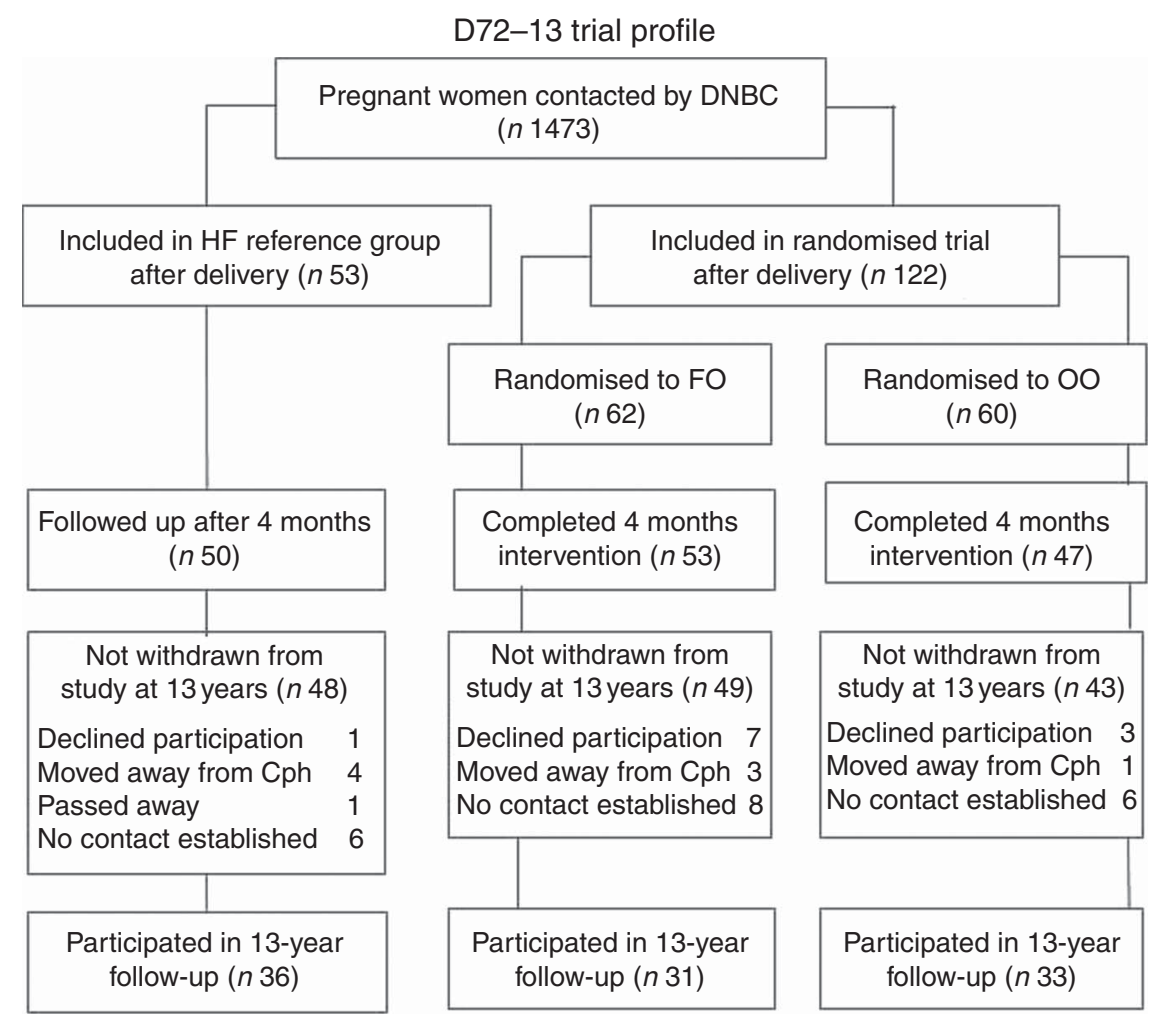

Fig. 1. Overview of the study flow. DNBC, Danish National Birth Cohort; HF, high-fish reference; FO, fish oil; OO, olive oil; Cph, Copenhagen. 
Table 1. Characteristics of the children in the three groups (Mean values and standard deviations or frequency $(n)$ )

\begin{tabular}{|c|c|c|c|c|c|c|}
\hline \multirow[b]{2}{*}{ At baseline } & \multicolumn{2}{|c|}{$\mathrm{HF}$} & \multicolumn{2}{|c|}{ ০০ } & \multicolumn{2}{|c|}{ FO } \\
\hline & Mean & $\mathrm{SD}$ & Mean & $\mathrm{SD}$ & Mean & SD \\
\hline$n(\mathrm{M}: \mathrm{F})$ & \multicolumn{2}{|c|}{$18: 18$} & \multicolumn{2}{|c|}{$16: 17$} & \multicolumn{2}{|c|}{ 19:12 } \\
\hline Paternal height (m) & 1.81 & 0.15 & 1.80 & 0.07 & 1.81 & 0.05 \\
\hline Maternal height (m) & 1.71 & 0.07 & 1.70 & 0.05 & 1.66 & 0.05 \\
\hline \multicolumn{7}{|l|}{ Maternal education (\%) } \\
\hline$<10-12$ years (high school or less) & 33.3 & 12 & $30 \cdot 3$ & 10 & $25 \cdot 8$ & 8 \\
\hline $13-14$ years & 19.4 & 7 & $30 \cdot 3$ & 10 & 9.7 & 3 \\
\hline $15-16$ years & $22 \cdot 2$ & 8 & $21 \cdot 2$ & 7 & $45 \cdot 2$ & 14 \\
\hline$>17$ years (higher academic) & $25 \cdot 0$ & 9 & 18.2 & 6 & $19 . \overline{4}$ & 6 \\
\hline Maternal age at delivery (years) & $32 \cdot 2$ & 3.9 & 30.5 & 3.8 & 30.9 & 3.9 \\
\hline Parity (\% primiparous) & \multicolumn{2}{|c|}{38.9} & \multicolumn{2}{|c|}{48.5} & \multicolumn{2}{|c|}{43.3} \\
\hline Gestational length (weeks) & $40 \cdot 1$ & $1 \cdot 2$ & $40 \cdot 1$ & $1 \cdot 2$ & $40 \cdot 3$ & 1.0 \\
\hline Birth weight (kg) & 3.58 & 0.47 & 3.54 & 0.45 & 3.64 & 0.45 \\
\hline Birth length $(\mathrm{cm})$ & 52.4 & 1.9 & $52 \cdot 2$ & $2 \cdot 1$ & 52.6 & $2 \cdot 3$ \\
\hline \multicolumn{7}{|l|}{ After randomisation } \\
\hline Breast-feeding duration (months) & 9.6 & $4 \cdot 1$ & $9 \cdot 6$ & 4.6 & 8.4 & $4 \cdot 1$ \\
\hline Breast milk DHA at 4 months (FA\%) & 0.73 & 0.35 & 0.41 & 0.21 & 1.29 & 0.41 \\
\hline Infant erythrocyte DHA at 4 months (FA\%) & 6.9 & $2 \cdot 2$ & 5.8 & $2 \cdot 0$ & 7.9 & $3 \cdot 1$ \\
\hline Child age at follow-up (years) & 13.5 & 0.4 & 13.4 & 0.4 & 13.5 & 0.4 \\
\hline
\end{tabular}

$\mathrm{HF}$, high-fish reference; OO, olive oil; FO, fish oil; M, male; F, female; FA\%, weight percentage of total fatty acids.

between the two groups was no longer significant after adjustment for puberty $(2 \cdot 4(95 \%$ CI $0.5,5 \cdot 3) \mathrm{cm}, P=0 \cdot 101)$. Overall, menarche had occurred in $66 \%$ of the girls, with no difference between the two randomised groups $(P=0 \cdot 31)$. None of the other anthropometric or body composition variables differed between the two randomised groups at 13 years of age or showed sex $\times$ group interactions (Table 2 ).

Dose-response analysis showed no clear negative association between maternal erythrocyte DHA at the end of the intervention period and height of the children at 13 years of age (all children included and adjusted for sex, age, mean parental height and breast-feeding: $\beta=-0.5(95 \%$ CI $-1 \cdot 2,0 \cdot 2) \mathrm{cm} / \mathrm{FA} \%, P=0 \cdot 17$, $n$ 98) (online Supplementary Fig. S1).

\section{Blood pressure}

The SBP, DBP and MAP of the children at 13 years did not differ between the randomised groups with the sexes combined, but a significant sex $\times$ group interaction was present for both DBP and MAP (Table 2). Analysis of FO $v$. OO adjusted for age and height in boys and girls separately showed a $3.9(95 \% \mathrm{CI} 0 \cdot 2,7 \cdot 5) \mathrm{mmHg}$ $(P=0.041)$ higher DBP and 3.6 (95\% CI $0.2,7.0) \mathrm{mmHg}$ ( $P=0.040)$ higher MAP with FO compared with $\mathrm{OO}$ in the boys and no differences in the girls. The difference in boys did not disappear after adjustment for puberty or physical activity (MAP difference adjusted for both: 4.9 (95\% CI $1.0,8.9) \mathrm{mmHg}$, $P=0 \cdot 017, n$ 27)

The sex-specific effect on MAP was supported by an interaction $(P=0.096)$ in the dose-response analysis (all children included), and the sex-specific analysis indicated a positive association between maternal erythrocyte DHA and MAP in boys $(\beta=0.7(95 \% \mathrm{CI}-0 \cdot 1,1.5) \mathrm{mmHg} / \mathrm{FA} \%, P=0.088, n 53)$, but not in girls $(\beta=-0.3$ (95\% CI $-1.1,0.6) \mathrm{mmHg} / \mathrm{FA} \%$, $P=0.52, n$ 45) (Fig. 3). The association between maternal erythrocyte DHA and DBP in boys was weaker $(\beta=0.7$ (95\% CI $-0 \cdot 2,1 \cdot 7) \mathrm{mmHg} / \mathrm{FA} \%, P=0 \cdot 110, n 53)$ than that for MAP.

\section{Discussion}

This follow-up study showed that adolescents of mothers who were randomised to FO supplementation during lactation were shorter than those whose mothers received OO supplements, an association that seemed to be driven by slower pubertal maturation. We also found a sex-specific effect of maternal FO supplementation on DBP and MAP, driven by a higher blood pressure in the boys from the FO group compared with the OO group and no difference in the girls. The effect of the intervention on blood pressure was independent of the effect on height and to some extent supported by dose-response associations with maternal DHA status at the end of the intervention period.

A recent Cochrane meta-analysis based on three trials concluded that maternal FO supplementation during pregnancy and lactation did not affect child weight and length up to 2 years of age and beyond ${ }^{(31)}$, although this was indicated in an earlier version of this analysis based on fewer trials but more participants $^{(32)}$. To our knowledge, the present study is the first study to investigate potential effects of early n-3 LCPUFA supplementation on growth during puberty, a time period with very high growth rates and major changes in body composition. The observation of shorter stature of children in the FO group at 13 years of age in the present study is in line with a previously observed negative association between erythrocyte DHA concentration of FO-supplemented mothers in the third trimester of pregnancy and child height at 6 years of age ${ }^{(33)}$. In contrast, others have found increased length at 6 years of age in a trial with LCPUFA-enriched infant formula ${ }^{(34)}$. Interestingly, a recent, genome-wide association analysis showed associations between certain SNP in the genes encoding for the fatty acid desaturases 
Table 2. Anthropometrics and blood pressure at 13 years of age in children of the three groups ${ }^{\star}$

(The raw data are given as mean values and standard deviations with $n$ in parenthesis if different from column $n$. The results from the statistical comparisons of the two randomised groups (olive oil (OO) and fish oil (FO)) performed by ANCOVA with inclusion of a sex $\times$ group interaction term are given as mean differences with their standard errors and $P$ values)

\begin{tabular}{|c|c|c|c|c|c|c|c|c|c|c|c|c|c|c|c|c|c|c|}
\hline & \multicolumn{4}{|c|}{$\mathrm{HF}$} & \multicolumn{4}{|c|}{$\mathrm{OO}$} & \multicolumn{4}{|c|}{ FO } & \multirow[b]{3}{*}{$P_{\text {group } x \text { sex }}$} & \multirow{2}{*}{\multicolumn{2}{|c|}{ FO v. OO (sex and age adjusted) }} & \multirow{2}{*}{\multicolumn{3}{|c|}{ FO v. OO (with additional adjustments)† }} \\
\hline & \multicolumn{2}{|c|}{ Boys } & \multicolumn{2}{|c|}{ Girls } & \multicolumn{2}{|c|}{ Boys } & \multicolumn{2}{|c|}{ Girls } & \multicolumn{2}{|c|}{ Boys } & \multicolumn{2}{|r|}{ Girls } & & & & & & \\
\hline & Mean & SD & Mean & SD & Mean & SD & Mean & SD & Mean & SD & Mean & SD & & Mean & $P$ & Mean & $\mathrm{SE}$ & $P$ \\
\hline$n$ & 18 & & 18 & & 16 & & 17 & & 19 & & & 12 & & & & & & \\
\hline Height (m) & 1.64 & 0.10 & 1.64 & 0.07 & 1.63 & 0.08 & 1.64 & 0.06 & 1.60 & 0.06 & 1.59 & $0.08(11)$ & 0.658 & -3.7 & 0.032 & -3.4 & 1.6 & 0.035 \\
\hline Weight (kg) & 47.0 & 9.5 & 49.9 & 9.5 & 48.7 & 7.2 & 48.2 & 9.1 & 48.5 & 7.8 & 48.0 & 11.4 & 0.997 & -0.6 & 0.779 & -0.9 & 2.0 & $\begin{array}{l}0.035 \\
0.664\end{array}$ \\
\hline $\mathrm{BMI}\left(\mathrm{kg} / \mathrm{m}^{2}\right)$ & 17.5 & 2.8 & 18.4 & 3.3 & 18.3 & 2.0 & 18.0 & 3.3 & 18.8 & 2.2 & $18 \cdot 7$ & 3.4 & 0.879 & $\begin{array}{r}-0.0 \\
0.5\end{array}$ & 0.460 & $\begin{array}{r}-0.9 \\
0.9\end{array}$ & 0.7 & 0.215 \\
\hline Fat mass indexł & 1.97 & 1.26 & 3.63 & 2.02 & 2.43 & 1.19 & 3.61 & 1.71 & 2.81 & 1.26 & 3.94 & 2.53 & 0.960 & 0.4 & 0.423 & 0.5 & 0.4 & 0.258 \\
\hline Body fat $\% \ddagger$ & 10.7 & 5.9 & 18.9 & 6.4 & 12.9 & 5.2 & 19.2 & 7.0 & 14.6 & 5.0 & 19.7 & 8.8 & 0.716 & 1.1 & 0.496 & 1.6 & 1.7 & 0.356 \\
\hline Sum of skinfolds (mm) & 8.1 & $2 \cdot 2$ & 11.4 & 4.8 & 8.7 & $2 . \overline{7}$ & 11.7 & 2.8 & 9.1 & 3.0 & 11.6 & 5.0 & 0.822 & 0.1 & 0.917 & 0.3 & 0.9 & 0.753 \\
\hline Waist circumference $(\mathrm{cm})$ & 68.7 & 8.0 & 71.7 & 7.2 & 71.2 & 4.5 & 69.9 & 5.9 & 69.8 & 5.9 & 69.6 & 10.7 & 0.767 & $-1 \cdot 2$ & 0.492 & -0.6 & 1.7 & 0.726 \\
\hline $\mathrm{DBP}(\mathrm{mmHg})$ & 61 & 8 & 63 & 5 & 61 & 6 & 65 & 5 & 64 & 4 & 63 & 5 & 0.022 & Boys: 3.6 (SE 1.7) & 0.048 & 3.9 & 1.8 & 0.041 \\
\hline $\mathrm{SBP}(\mathrm{mmHg})$ & 107 & 8 & 105 & 5 & 109 & 10 & 108 & 6 & 110 & 6 & 104 & 9 & 0.223 & $\begin{array}{l}\text { Girls:-2.3 (SE } 1.9) \\
-1.4\end{array}$ & $\begin{array}{l}0.226 \\
0.467\end{array}$ & $\begin{array}{l}-2.1 \\
-0.6\end{array}$ & $\begin{array}{l}2.0 \\
1.7\end{array}$ & $\begin{array}{l}0.298 \\
0.742\end{array}$ \\
\hline $\operatorname{MAP}(\mathrm{mmHg})$ & 76.6 & 6.6 & 76.8 & 4.4 & 76.8 & $6 \cdot 4$ & 79.5 & 3.8 & 79.5 & $\begin{array}{l}3.4 \\
3.4\end{array}$ & 76.6 & $5 \cdot 7$ & 0.030 & Boys: 2.6 (SE 1.7 ) & 0.137 & $\begin{array}{r}-0.0 \\
3 \cdot 6\end{array}$ & 1.7 & 0.040 \\
\hline & & & & & & & & & & & & & & Girls: -2.9 (SE 1.8) & 0.126 & $-2 \cdot 3$ & 1.9 & 0.248 \\
\hline
\end{tabular}

HF, high fish; DBP, diastolic blood pressure; SBP, systolic blood pressure; MAP, mean arterial pressure.

* Data for the high fish group are included as reference.

† The model for height was adjusted for sex, age and mean parental height; models for body weight and body composition outcomes were adjusted for sex, age and puberty; and the blood pressure models were adjusted for sex, age and height.

‡ Based on bioimpedance.
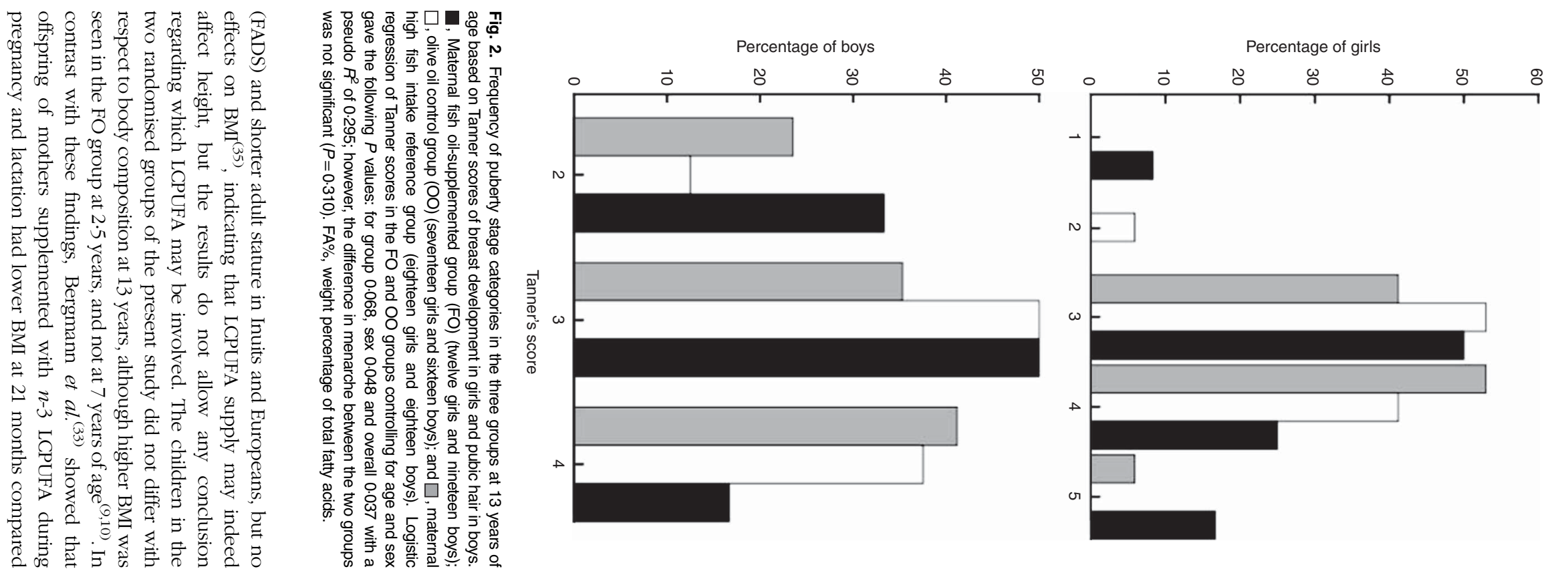


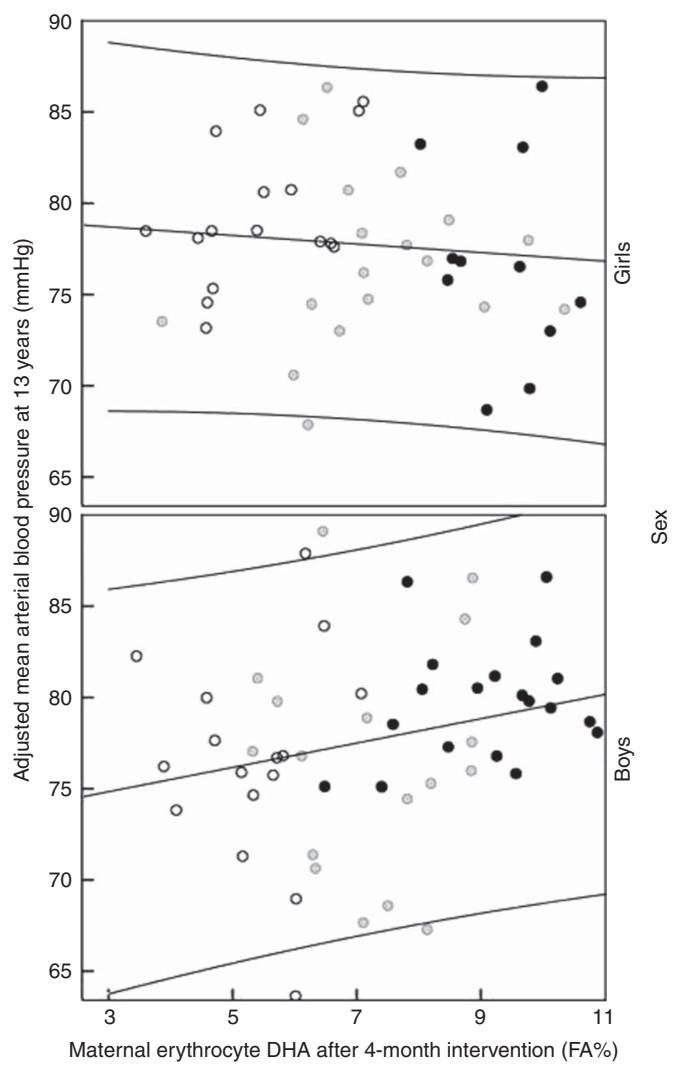

Fig. 3. Association between mean arterial blood pressure at 13 years of age adjusted for age, height and breast-feeding and maternal erythrocyte DHA (erythrocyte DHA) at the end of the intervention period in girls and boys in the three groups $(\bigcirc$, maternal fish oil-supplemented group; $\bigcirc$, olive oil control group; maternal high fish intake reference group). The regression lines are given with $95 \%$ prediction interval. FA\%, weight percentage of total fatty acids. Girls $R^{2}$ linear $=0.009$; boys $R^{2}$ linear $=0.057$.

with the control group, but this difference in BMI was also no longer evident at 6 years. In line with this, a recent systematic review states that the results regarding early supply of $n-3$ LCPUFA and later adiposity in children are inconsistent ${ }^{(2)}$.

In adolescence, height is pronouncedly affected by pubertal stage, and the effect on height at 13 years was no longer significant after adjustment for puberty, indicating that the difference in height may be due to a slower pubertal maturation with early FO supplementation. A tendency towards later onset of puberty after maternal FO supplementation was indicated, although not statistically significant, and the study may be underpowered to truly detect such self-reported developmental differences. A delay in puberty could be due to a different growth pattern, as BMI decreased from 2.5 to 7 years of age in the FO group, whereas it increased in the OO group, and it could be speculated that this could reflect a later adiposity rebound in the FO group. In a Danish cohort study, a delay in adiposity rebound was observed in children of mothers with a breast milk DHA content in the upper quintile compared with the other quintiles ${ }^{(14)}$. No sex-specific effects on growth or body composition were seen in our previous FO trial in infants ${ }^{(36)}$, which, however, showed increased serum insulin-like growth factor (IGF)-1 concentrations after FO supplementation in boys only ${ }^{(37)}$. In a $\mathrm{FO}$ trial in adolescent boys, we also found a positive association between changes in erythrocyte $n-3$ LCPUFA and changes in plasma IGF-1 during the intervention $^{(38)}$. To our knowledge, long-term sex-specific effects of early $n$-3 LCPUFA supplementation on growth or body composition have not been investigated previously, but our data in the present follow-up study do not indicate any interaction between $n$-3 LCPUFA supplementation and sex on the anthropometric outcomes.

The observed higher blood pressures in 13-year-old boys, but not in girls, exposed to breast milk from FO-supplemented $v$. OO-supplemented mothers during infancy confirm the previously observed effect at 7 years in this study population and may indicate that early $n$-3 LCPUFA intake can have effects that persist into puberty. The observed results are in line with our previous findings in cross-sectional studies of 8-11-year-old Danish children, which showed positive associations between whole-blood $n$-3 LCPUFA and blood pressure in boys only $^{(22,23)}$. The studies are, however, in contrast with previous observations from randomised trials in adults, which generally show the blood pressure-reducing effects of $n-3$ LCPUFA $^{(39)}$. Furthermore, Forsyth et al. ${ }^{(17)}$ have demonstrated that infants fed formula enriched with $n$-3 LCPUFA had lower blood pressure at age 6 years compared with children receiving formula with no $n-3$ LCPUFA and a low $\alpha$-linolenic acid content, a finding that is supported by animal studies ${ }^{(15,16)}$. In line with this, a recent observational study showed that children who received breast milk with a high content of $n$ - 3 LCPUFA had lower blood pressure at 12 years of age compared with children who were never breast-fed ${ }^{(40)}$. The lower height in the FO group in the present study could not explain the increased blood pressure, as the effect was found regardless of adjustment for height, and furthermore blood pressure is generally positively associated with height in children ${ }^{(41)}$.

Previous studies that have investigated potential sex-specific effects of FO supplementation in randomised trials seem to indicate that FO supplementation dampens sex differences particularly in relation to blood pressure ${ }^{(42)}$ - but also cognitive outcomes ${ }^{(43)}$. Moreover, sex has been shown to be an important determinant of LCPUFA status in both rats and humans ${ }^{(38)}$. This is likely mediated by the influence of sex hormones on the enzymatic synthesis of LCPUFA, resulting in a higher content of DHA in, for example, the liver and plasma in females compared with males ${ }^{(44,45)}$. In contrast, data from the present study did not show sex differences in DHA status at 13 years, but fatty acid analyses were also only performed in a subgroup of the children. It is unknown whether $n-3$ LCPUFA intake can affect sex hormone levels, but this may hypothetically explain why the children of the FO-supplemented mothers tended to have delayed pubertal maturation than the children of the OO-supplemented mothers. More studies are needed to elucidate this further.

The difference in puberty could be a chance finding, as one major limitation of the present study is the relatively low sample size. Insufficient statistical power could particularly be an issue in the sex-specific analyses. However, to our knowledge, this is the only study that followed-up children from a randomised controlled trial with early $n$ - 3 LCPUFA supplementation at the age of puberty. Furthermore, participation (70\%) was relatively high 
compared with what could be anticipated in such a long-term study, and the participating children were reasonably representative of the initial trial population. However, owing to the selective nature of the recruiting procedure for the original trial, the children were generally not representative for the overall Danish population, but this is expected in this type of trial. Puberty was self-assessed by the children in order to respect their sexual privacy. Although the line drawings used to support the children's puberty ratings have been validated previously ${ }^{(46)}$, this may have given rise to some imprecision. The study could have been strengthened by the measurement of serum IGF-1 to support the growth measurements, but venous blood sampling was unfortunately not possible, and we were only able to get fingertip prick blood samples from half of the children, as ethics approval for this procedure was delayed. We did not manage to get blood from all the infants at the end of the intervention, and we have therefore used maternal erythrocyte DHA in the doseresponse analyses adjusted for the degree of breast-feeding. We have also data on breast milk fatty acid composition, but this fluctuates from day to day, and thus would not provide a stable measure of the infants DHA intake during the intervention. In this study, we performed a number of statistical tests, but we did not apply, for example, Bonferroni correction, first of all because the study is exploratory in its nature, and also because many of the outcomes were associated, and a Bonferroni correction would be overly conservative.

The potential delay in children's sexual maturation and thereby a reduction in height at 13 years indicated in this study could have long-term effects on final height. However, some studies indicate that delayed puberty is associated with an increase in adult height caused by a longer period of pre-pubertal growth ${ }^{(47)}$. Furthermore, fish intake is high and FO supplementation in infancy is common in Nordic countries, especially in Norway, and the populations in these countries are among the tallest in the world ${ }^{(48)}$. However, the higher blood pressure among boys from the maternal FO group is consistent with results from the 7-year follow-up. It may be an adverse effect if it tracks into adulthood, as increased blood pressure is one of the major determinants of CVD and stroke. The children are as mentioned not representative of the Danish population, but if confirmed the findings may reflect biological effects, and thus may be transferable to children in general. It is therefore crucial to perform long-term follow-up studies on other early $n$-3 LCPUFA supplementation trials, and ideally these studies should also explore the potential for sex specificity.

In conclusion, the results of this study showed that early n-3 LCPUFA intake was associated with lower height in early adolescence and with increased DBP in boys only. However, due to the small sample size of the study, these novel findings need to be confirmed in larger randomised controlled trials.

\section{Acknowledgements}

The authors thank Christian Lundtofte, who voluntarily helped with data collection, and all the kids and their parents who participated in the study.

The initial randomised controlled trial was supported by FØTEK, The Danish Research and Development Program for Food and Technology and BASF Aktiengesellschaft.
The randomised controlled trial was planned and designed by L. L. collaboration with K. F. M., and the 13-year follow-up was initiated by L. L., M. S. N. and C. T. D., S. E. E. and M. S. N. completed all the data collection; M. F. H. and K. D. S. were responsible for the analysis of physical activity recordings and whole-blood fatty acids, respectively. L. L. and S. E. E. performed the statistical analysis and interpretation, as well as drafted the manuscript. All the authors commented on the manuscript and approved the its final version.

L. L., S. E. E., M. F. H., M. S. N., S. F. O., K. D. S., K. F. M. and C. T. D. declare no conflicts of interest.

\section{Supplementary material}

For supplementary material/s referred to in this article, please visit https://doi.org/10.1017/S0007114516004293

\section{References}

1. Lauritzen L, Hansen HS, Jørgensen MH, et al. (2001) The essentiality of long-chain $n-3$ fatty acids in relation to development and function of the brain and retina. Prog Lipid Res 40, 1-94.

2. Voortman T, van den Hooven EH, Braun KVE, et al. (2015) Effects of polyunsaturated fatty acid intake and status during pregnancy, lactation, and early childhood on cardiometabolic health: a systematic review. Prog Lipid Res 59, 67-87.

3. Harsløf LBS, Larsen LH, Ritz C, et al. (2013) FADS genotype and diet are important determinants of DHA status: a cross-sectional study in Danish infants. Am J Clin Nutr 97, 1403-1410.

4. Owen CG, Martin RM, Whincup PH, et al. (2005) The effect of breastfeeding on mean body mass index throughout life: a quantitative review of published and unpublished observational evidence. Am J Clin Nutr 82, 1298-1307.

5. Innis SM (2011) Metabolic programming of long-term outcomes due to fatty acid nutrition in early life. Matern Child Nutr 7, 112-123.

6. Martin RM, Gunnell D \& Smith GD (2005) Breastfeeding in infancy and blood pressure in later life: systematic review and meta-analysis. Am J Epidemiol 161, 15-26.

7. Kim HK, Della-Fera M, Lin J, et al. (2006) Docosahexaenoic acid inhibits adipocyte differentiation and induces apoptosis in 3T3-L1 preadipocytes. J Nutr 136, 2965-2969.

8. Ailhaud G, Massiera F, Weill P, et al. (2006) Temporal changes in dietary fats: role of $n$ - 6 polyunsaturated fatty acids in excessive adipose tissue development and relationship to obesity. Prog Lipid Res 45, 203-236.

9. Lauritzen L, Hoppe C, Straarup EM, et al. (2005) Maternal fish oil supplementation in lactation and growth during the first 2.5 years of life. Pediatr Res 58, 235-242.

10. Asserhøj M, Nehammer S, Matthiessen J, et al. (2009) Maternal fish oil supplementation during lactation may adversely affect long-term blood pressure, energy intake, and physical activity of 7-year-old boys. J Nutr 139, 298-304.

11. Bergmann RL, Bergmann KE, Haschke-Becher E, et al. (2007) Does maternal docosahexaenoic acid supplementation during pregnancy and lactation lower BMI in late infancy? J Perinat Med 35, 295-300.

12. Helland IB, Smith L, Blomen B, et al. (2008) Effect of supplementing pregnant and lactating mothers with $n-3$ very-long-chain fatty acids on children's IQ and body mass index at 7 years of age. Pediatrics 122, E472-E479.

13. Rolland-Cachera MF, Deheeger M, Bellisle F, et al. (1984) Adiposity rebound in children - a simple indicator for predicting obesity. Am J Clin Nutr 39, 129-135. 
14. Pedersen L, Lauritzen L, Brasholt M, et al. (2012) Polyunsaturated fatty acid content of mother's milk is associated with childhood body composition. Pediatr Res 72, 631-636.

15. Wyrwoll CS, Mark PJ, Mori TA, et al. (2006) Prevention of programmed hyperleptinemia and hypertension by postnatal dietary omega-3 fatty acids. Endocrinology 147, 599-606.

16. Armitage JA, Pearce AD, Sinclair AJ, et al. (2003) Increased blood pressure later in life may be associated with perinatal n-3 fatty acid deficiency. Lipids 38, 459-464.

17. Forsyth JS, Willatts P, Agostoni C, et al. (2003) Long-chain polyunsaturated fatty acid supplementation in infant formula and blood pressure in later childhood: follow up of a randomised controlled trial. BMJ 326, 953.

18. Ayer JG, Harmer JA, Xuan W, et al. (2009) Dietary supplementation with $n$-3 polyunsaturated fatty acids in early childhood: effects on blood pressure and arterial structure and function at age 8 y. Am J Clin Nutr 90, 438-446.

19. Rytter D, Christensen JH, Bech BH, et al. (2012) The effect of maternal fish oil supplementation during the last trimester of pregnancy on blood pressure, heart rate and heart rate variability in the 19 -year-old offspring. BrJ Nutr $\mathbf{1 0 8}$, $1475-1483$.

20. Lauritzen L, Jørgensen MH, Mikkelsen TB, et al. (2004) Maternal fish oil supplementation in lactation: effect on visual acuity and $n-3$ fatty acid content of infant erythrocytes. Lipids 39, 195-206.

21. Harsløf LB, Damsgaard C, Hellgren L, et al. (2014) Effects on metabolic markers are modified by PPARG2 and COX2 polymorphisms in infants randomized to fish oil. Genes Nutr 9, 1-11.

22. Damsgaard CT, Stark KD, Hjorth MF, et al. (2013) n-3 PUFA status in school children is associated with beneficial lipid profile, reduced physical activity and increased blood pressure in boys. Br J Nutr 110, 1304-1312.

23. Damsgaard CT, Eidner MB, Stark KD, et al. (2014) Eicosapentaenoic acid and docosahexaenoic acid in wholeblood are differentially and sex-specifically associated with cardiometabolic risk markers in 8-11-year-old Danish children. PLOS ONE 9, e109368.

24. Makrides M (2013) DHA supplementation during the perinatal period and neurodevelopment: Do some babies benefit more than others? Prostaglandins Leukot Essent Fatty Acids 88, 87-90.

25. Ulbak J, Lauritzen L, Hansen HS, et al. (2004) Diet and blood pressure in 2.5-y-old Danish children. Am J Clin Nutr 79, 1095-1102.

26. Olsen J, Melby M, Olsen SF, et al. (2001) The Danish National Birth Cohort - its background, structure and aim. Scand J Public Health 29, 300-307.

27. Slaughter MH, Lohman TG, Boileau RA, et al. (1988) Skinfold equations for estimation of body fatness in children and youth. Hum Biol 60, 709-723.

28. Tanner JM (1962) Growth at Adolescence, 2nd ed. Oxford: Blackwell Scientific Publishers.

29. Metherel AH, Aristizabal Henao JJ \& Stark KD (2013) EPA and DHA levels in whole-blood decrease more rapidly when stored at $-20^{\circ} \mathrm{C}$ as compared with room temperature, 4 and $75^{\circ} \mathrm{C}$. Lipids 48, 1079-1091.

30. Bjerregaard AA, Tetens I, Olsen SF, et al. (2016) Reproducibility of a web-based FFQ for 13- to 15-year-old Danish adolescents. J Nutr Sci 5, e5.

31. Delgado-Noguera MF, Calvache JA, Cosp XB, et al. (2015) Supplementation with long-chain polyunsaturated fatty acids (LCPUFA) to breastfeeding mothers for improving child growth and development. The Cochrane Database of
Systematic Reviews, issue 7, CD007901. http://onlinelibrary. wiley.com/doi/10.1002/14651858.CD007901.pub3/full

32. Delgado-Noguera MF, Calvache JA \& Cosp XB (2010) Supplementation with long-chain polyunsaturated fatty acids (LCPUFA) to breastfeeding mothers for improving child growth and development. The Cochrane Database of Systematic Reviews, issue 12, CD007901. http://onlinelibrary. wiley.com/doi/10.1002/14651858.CD007901.pub2/full

33. Bergmann RL, Bergmann KE, Richter R, et al. (2012) Does docosahexaenoic acid (DHA) status in pregnancy have any impact on postnatal growth? Six-year follow-up of a prospective randomized double-blind monocenter study on lowdose DHA supplements. J Perinat Med 40, 677-684.

34. Currie LM, Tolley EA, Thodosoff JM, et al. (2015) Long-chain polyunsaturated fatty acid supplementation in infancy increases length- and weight-for-age but not BMI to 6 years when controlling for effects of maternal smoking. Prostaglandins Leukot Essent Fatty Acids 98, 1-6.

35. Fumagalli M, Moltke I, Grarup N, et al. (2015) Greenlandic Inuit show genetic signatures of diet and climate adaptation. Science 349, 1343-1347.

36. Andersen AD, Michaelsen KF, Hellgren LI, et al. (2011) A randomized controlled intervention with fish oil versus sunflower oil from 9 to 18 months of age: exploring changes in growth and skinfold thicknesses. Pediatr Res $\mathbf{7 0}$, 368-374.

37. Damsgaard CT, Harsløf LBS, Andersen AD, et al. (2016) Fish oil supplementation from 9 to 18 months of age affects the insulin-like growth factor axis in a sex-specific manner in Danish infants. Br J Nutr 115, 782-790.

38. Damsgaard CT, Mølgaard C, Matthiessen J, et al. (2012) The effects of $n$-3 long-chain polyunsaturated fatty acids on bone formation and growth factors in adolescent boys. Pediatr Res $\mathbf{7 1}$, 713-719.

39. Miller PE, Van Elswyk M \& Alexander DD (2014) Long-chain omega-3 fatty acids eicosapentaenoic acid and docosahexaenoic acid and blood pressure: a meta-analysis of randomized controlled trials. Am J Hypertens 27, 885-896.

40. van Rossem L, Wiiga AH, de Jongste JC, et al. (2012) Blood pressure in 12-year-old children is associated with fatty acid composition of human milk - the prevention and incidence of asthma and mite allergy birth cohort. Hypertension 60, 1055-1060.

41. Neuhauser HK, Thamm M, Ellert U, et al. (2011) Blood pressure percentiles by age and height from non-overweight children and adolescents in Germany. Pediatrics 127, E978-E988.

42. Lauritzen L, Brambilla P, Mazzocchi A, et al. (2016) DHA effects in brain development and function. Nutrients $\mathbf{8}, 6$.

43. Makrides M, Gibson RA \& Mcphee AJ (2010) Effect of DHA supplementation during pregnancy on maternal depression and neurodevelopment of young children: a randomized controlled trial. JAMA 304, 1675-1683.

44. Childs CE, Romeu-Nadal M, Burdge GC, et al. (2010) The polyunsaturated fatty acid composition of hepatic and plasma lipids differ by both sex and dietary fat intake in rats. $J$ Nutr 140, 245-250.

45. Kitson A, Stroud C \& Stark K (2010) Elevated production of docosahexaenoic acid in females: potential molecular mechanisms. Lipids 45, 209-224.

46. Morris NM \& Udry JR (1980) Validation of a self-administered instrument to assess stage of adolescent development. $J$ Youth Adolesc 9, 271-280.

47. Wellens R, Malina RM, Roche AF, et al. (1992) Body size and fatness in young adults in relation to age at menarche. $\mathrm{Am} \mathrm{J}$ Hum Biol 4, 783-787.

48. NCD Risk Factor Collaboration (2016) A century of trends in adult human height. eLife 5, e13410. 